\title{
SELF-ADMINISTERED INTRAVESICAL HYALURONIC ACID IMPROVES SYMPTOMS AND QUALITY OF LIFE IN A PATIENT-CENTRED APPROACH TO BLADDER PAIN SYNDROME MANAGEMENT
}

Mark O. Kitchen ${ }^{1,2,3}$, Helen Thursby ${ }^{1}$, Monica Taylor ${ }^{1}$, Kay Willard ${ }^{1}$, Tina Mistry-Pain ${ }^{1}$

${ }^{1}$ Urology Department, University Hospitals of North Midlands NHS Trust, Staffordshire, UK

${ }^{2}$ Institute for Science and Technology in Medicine, Keele University, Staffordshire, UK

${ }^{3}$ Institute for Applied Clinical Science, Keele University, Staffordshire, UK

Corresponding author: m.o.kitchen@keele.ac.uk

Submitted: August 18, 2019. Accepted: September 13, 2019. Published: October 28, 2019.

\begin{abstract}
\section{Background and Objectives}

Bladder pain syndrome (BPS) is an uncommon and potentially debilitating spectrum of chronic pain typically accompanied by lower urinary tract symptoms. Intravesical hyaluronic acid (HA) is a commonly used treatment option, but requires multiple follow-up clinic appointments. We introduced a novel patient-led 'athome' pathway of self-administered HA treatment to reduce the number hospital visits required by patients.

We assessed and compared patient-reported outcome measures (PROMS) from patients receiving nurse-administered 'in-hospital' and patient-led self-administered 'at-home' intravesical HA (Cystistat ${ }^{\circledR}$ and Hyacyst ${ }^{\circledR}$, respectively). Secondary outcome measures included differences between waiting times for treatment, frequency of treatments, number of clinician and nurse clinic appointments, and estimated financial costs.
\end{abstract}

\section{Patients and Methods}

Sixty consecutive patients commencing intravesical HA for BPS symptoms between 1st January 2016 and 31 st March 2019 were included. O'Leary-Sant Interstitial Cystitis Symptom Index questionnaires were completed prior to, and following, six treatments. Relevant clinical and demographic data were also collected.

\section{Results}

Overall, $76.6 \%$ of the patients had improvement in symptoms after HA treatment. Mean O'Leary-Sant questionnaire symptom and problem scores were significantly improved following HA treatment (11.8 (range $6-17$ ) to 8.5 (range 4-13) ( $p=0.00005)$ and 11.4 (range 4-16) to 7.9 (range 4-14) ( $p=0.0002$ ), respectively. There were no significant differences in symptom improvements between patients on either pathway.

Mean waiting time for treatment and number of treatments were lower in the patient-led pathway, and number of hospital visits was significantly lower in the patient-led at home pathway. The patient-led pathway could 'save' approximately 76 nurse clinic and 11 outpatient clinic appointments per year, and confer cost-savings of more than $£ 1,500$ per patient, per year. 


\section{Conclusions}

Our case series analyses suggest that patient-led 'at-home' intravesical HA administration (Hyacyst巴) is acceptable to patients and confers similar symptomatic benefit to 'in-hospital' nurse-led HA (Cystistat $\left.{ }^{\circledR}\right)$. In addition, it appears that BPS sufferers established on the patient-led pathway require fewer HA treatments and fewer hospital visits, and as such, the patient-led pathway may also confer financial cost savings, and relieve some pressures on clinic appointment availability.

Key words:_Bladder pain syndrome; intravesical hyaluronic acid; patient-reported outcome measures

Bladder pain syndrome (BPS) is an uncommon, heterogeneous, and potentially debilitating spectrum of chronic pain, perceived by the sufferer to originate from the bladder. ${ }^{1}$ BPS predominantly affects women with peak age of onset at $45-60$ years, and has an estimated worldwide prevalence of 3-6\%., ${ }^{2,3}$ In addition to chronic pain, BPS is typically associated with lower urinary tract symptoms such as urgency, frequency, dysuria, nocturia (increasing with age of patient), and pain on filling and/or voiding, and non-urinary symptoms such as genital pain and dyspareunia. ${ }^{1,3,4}$ BPS is associated with a significant reduction in quality of life, and may impact psychological (low mood, depression, anxiety and sleep disturbance), relationship and sexual, and work/employment functions. ${ }^{4-6}$

Although the aetiology of BPS is incompletely understood, it is thought that loss of glycosaminoglycans (GAGs) is a key step in pathogenesis. ${ }^{7,8}$ GAG loss may contribute to bladder mucosal surface disruption, exposing the underlying urothelium to noxious stimuli; the resultant immune and inflammatory responses causing the range of symptoms observed..$^{7-9}$ Multiple studies have demonstrated that the exogenous administration of hyaluronic acid (HA), a member of the GAG family, improves patient outcomes in BPS. ${ }^{10-12}$ Therefore, and following lifestyle/fluid advice and holistic management steps, ${ }^{13,14}$ the mainstay of current treatment at our University Teaching Hospital is GAG replacement with intravesical HA (Cystistat ${ }^{\circledR} 40 \mathrm{mg}$ ).

Historically, we used a predefined schedule of nurse-led clinic visits for HA instillation. However, feedback from patients and increased demand for our nurse-led clinics (particularly for intravesical therapies for bladder cancer patients) necessitated the introduction of a novel patient-led 'at-home' pathway, comprising teaching intermittent self-catheterisation
(ISC) and self-administration of HA (Hyacyst ${ }^{\circledR}$ prefilled syringe $40 \mathrm{mg}$ ).

As a novel and unique treatment pathway, we sought to assess and compare patient-reported outcome measures (PROMS) from patients receiving scheduled nurse-administered 'in-hospital' and patient-led selfadministered intravesical HA. As secondary outcome measures, we assessed the differences between treatment pathways in waiting times for treatment, frequency of treatments, number of clinician and nurse clinic appointments (hospital visits) required, and potential financial costs on a per-patient basis.

\section{PATIENTS AND METHODS}

All patients commencing intravesical hyaluronic acid (HA) treatment for Bladder Pain Syndrome (BPS) symptoms between 1st January 2016 and 31st March 2019 were included for analyses as a consecutive case series $(n=60)$. All patients were invited to complete O'Leary-Sant Interstitial Cystitis Symptom Index questionnaires ${ }^{15}$ (Supplemental Figure 1.) prior to, and following six intravesical treatments (in the absence of urinary tract infection). Where questionnaire data were not available, patient symptoms documented in medical notes were recorded. Clinical and demographic data were collected alongside the patient-reported outcome measures (PROMS). The number of treatments and number of hospital visits (either clinician or specialist nurse) were also recorded.

Microsoft Excel (Microsoft Corporation, USA, v16.0, 2016) and online Social Science Statistics software ${ }^{16}$ were used for statistical analyses. Paired-sample and standard $\mathrm{T}$ tests were used to compare pre- and post-treatment questionnaire scores, and differences between outcomes of nurse-led and patient-led pathways ( $p<0.05$ considered significant). 


\section{RESULTS}

\section{Patient Demographics}

A total of 60 patients were included. 55 (91.7\%) were female. Mean age was 50.4 years (range 18 to 86). Predominant symptoms were urinary tract infections $(n=29)$, pain $(n=24)$ and refractory bothersome lower urinary tract symptoms (LUTS) $(n=7)$.

Of the 60 patients included, 28 chose to start on the nurse-led pathway and 32 chose to start on the patient-led pathway. 14/28 successfully moved from the nurse-led Cystistat ${ }^{\circledR}$ to the patient-led at-home Hyacyst ${ }^{\circledR}$ pathway. Two patients were unsuccessful in moving to the patient-led pathway (did not want to self-catheterise), 6 patients declined self-administration as they had no benefit from HA treatment, 2 patients discontinued during pregnancy, and 4 patients had not yet been approached.

\section{Patient-Reported Outcome Measures Following Intravesical Hyaluronic Acid (HA) Treatment}

Completed pre- and post-treatment questionnaires were available for $31 / 60$ patients $(51.7 \%)$. As shown in Figure 1. (panel a.), mean IC symptom index (ICSI) scores pre-treatment were 11.8 (range 6-17), and 8.5 (range 4-13) following HA treatment ( $p=0.00005$ ). Mean IC problem index (ICPI) scores pre-treatment were 11.4 (range 4-16), and 7.9 (range 4-14) following HA treatment $(p=0.0002)$. Symptomatic improvement was reported/observed in 46/60 (76.6\%) patients overall.

Stratifying the ICSI further into component questions (Figure 1. panel b.), mean urgency scores (Question 1 (Q1)) were 3.5 and 2.5 pre- and post-treatment, respectively ( $p=0.001)$, frequency scores (Q2) were 3.3 and 2.4 pre- and post-treatment $(p=0.008)$, nocturia scores (Q3) were 2.0 and 1.7 pre- and post-treatment $(p=0.172)$, and pain and burning scores $(\mathrm{Q} 4)$ were 3.1 and 1.9 pre- and post-treatment $(p=0.00004)$.

Separating the ICPI (Figure 1. panel c.), mean frequency scores (Q1) were 3.1 and 2.1 pre- and post-treatment, respectively $(p=0.001)$, nocturia scores (Q2) were 2.1 and 1.5 pre- and post-treatment $(p=0.019)$, urgency scores (Q3) were 2.9 and 2.0 preand post-treatment ( $p=0.003$ ), and pain and burning scores $(\mathrm{Q} 4)$ were 3.3 and 2.3 pre- and post-treatment $(p=0.0003)$.

\section{Patient-Reported Outcome Measures of Nurse-Led and Patient-Led Pathways}

There were no differences in the nurse-led Cystistat ${ }^{\circledR}$ and the patient-led at-home Hyacyst ${ }^{\circledR}$ pathway populations (age, gender, comorbidity, predominant symptom or questionnaire scores).

As shown in Figure 2. (panel a.), in the nurse-led pathway patients, mean ICSI pre-treatment scores were 11.9 (range 8-17), and 8.2 (range 5-16) following treatment $(p=0.002)$, and mean ICPI scores were 11.6 (range 4-16) pre-treatment, and 7.6 (range $4-13)$ following treatment $(p=0.003)$. In the patient-led pathway, mean ICSI scores pre-treatment were 11.8 (range 6-17) and 8.7 (range 4-16) following treatment $(p=0.005)$, and mean ICPI scores were 11.3 (range 6-16) pre-treatment and 8.2 (range 4-14) following treatment $(p=0.001)$.

There was no significant difference in overall symptom or problem score reduction between the nurse-led and patient-led pathway ( $p=0.131)$.

Mean reduction in ICSI and ICPI scores for the nurse-led and patient-led pathways across all questions are shown in Figure 2. panels b. and c., respectively. Mean reduction in symptom scores for the nurse-led and patient-led pathways for urgency (Q1) were 1.3 and $0.8,(p=0.114)$, frequency (Q2) were 1.3 and 0.9 $(p=0.184)$, nocturia (Q3) were 0.4 and $0.1(p=0.134)$, and pain and burning (Q4) were 1.1 and $1.4(p=0.175)$, respectively. Mean reduction in problem scores for the nurse-led and patient-led pathways for frequency (Q1) were 1.4 and 0.7 , respectively $(p=0.068)$, nocturia (Q2) were 0.9 and $0.3(p=0.058)$, urgency (Q3) were 1.2 and $0.8(p=0.173)$, and pain and burning (Q4) were 0.9 and $1.2(p=0.198)$, respectively.

\section{Patient-Reported Outcome Measure Scores in Relation to Clinical and Demographic Factors}

Multivariate regression analyses suggested that patient age, gender, number of comorbidities, predominant symptom, and treatment type, were not independently associated with improvement in IC symptom or problem scores ( $p>0.05$, data not shown).

\section{Potential Implications of Establishing the Patient-Led Pathway for Intravesical $\mathrm{HA}$}

In addition to PROMS, we also evaluated other possible benefits to patients and to our service provision 
FIG. 1 IC symptom and problem questionnaire scores prior to and following intravesical hyaluronic acid. Panel a. shows the pre- and post-treatment O'Leary-Sant questionnaire symptom and problem scores. Pretreatment mean is represented by the solid blue bar; the post-treatment mean is represented by the solid green bar. Significant differences in scores are denoted by '*', where $\mathrm{p}<0.05$ by T Test. Panels $\mathrm{b}$. and c. show the pre- and post-treatment scores for the specific symptom areas that comprise the symptom and problem questionnaire scores, respectively. The blue bars represent mean pre-treatment score and the green bars represent post-treatment mean scores.

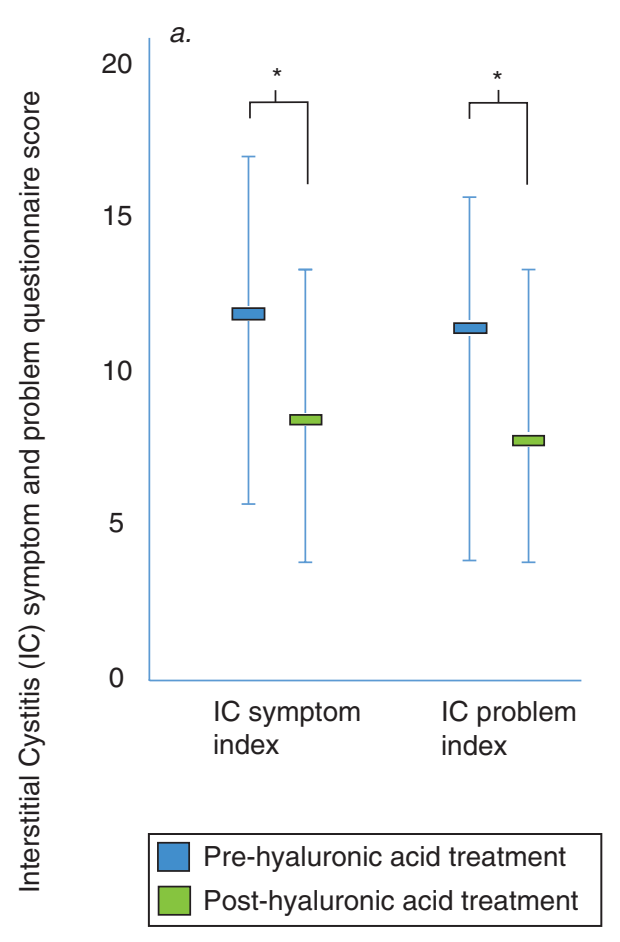

gained by using the patient-led pathway. As shown in Figure 3. (in the nurse- and patient-led pathways, respectively) average waiting time to start treatment was 16.7 and 14.9 weeks $(p=0.169)$ (panel a.); mean number of treatments were 12.0 and $10.4(p=0.062)$ (panel b.), mean number of nurse clinic appointments were 12.0 and 7.1 ( $p=0.0001)$ (panel c.), mean number of clinician outpatient appointments were 1.0 and 0.3 ( $p=0.001$ ) (panel d.), and estimated cost per patient was $£ 4,860$ and $£ 3,350$ (all per year).

\section{DISCUSSION}

Painful bladder syndrome (PBS) is associated with a broad range of symptoms and significant reduction
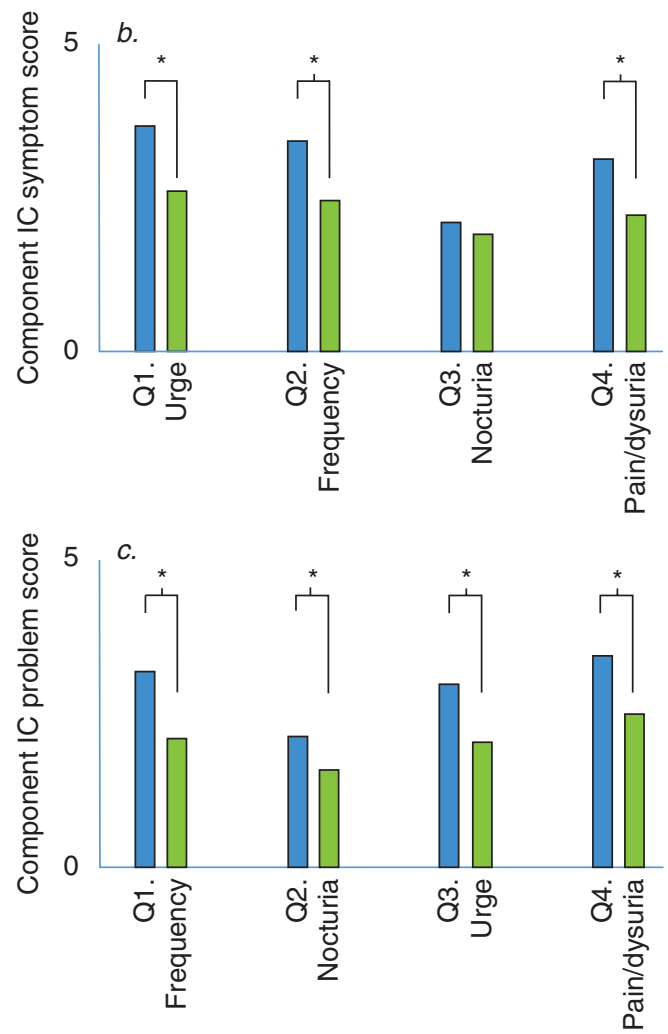

in quality of life. ${ }^{4-6}$ Although no treatment option provides reliable and significant improvements, exogenous hyaluronic acid (HA) administration has been shown to reduce symptom severity and improve quality of life for PBS patients. ${ }^{10-12}$

Hitherto, our BPS patients received regular nurse-led clinic appointments for intravesical HA instillations (Cystistat ${ }^{\circledR} 40 \mathrm{mg}$ )(once per week for 6 weeks, then monthly thereafter), with regular nurse and outpatient clinic review. Patient feedback regarding inconvenience of multiple hospital visits, combined with pressures on service provision, necessitated the introduction of an 'at-home' patient-led pathway, comprising selfcatheterisation teaching and self-administration of 
FIG. 2 IC symptom and problem questionnaire scores in the nurse-led and patient-led intravesical hyaluronic acid pathways.

Panel a. shows the pre- and post-treatment O'Leary-Sant questionnaire symptom and problem scores for the nurse-led and patient-led pathways. Pre-treatment mean is represented by the solid blue bars, the post-treatment mean is represented by the solid yellow bars (nurse-led) and purple bars (patient-led). Significant differences in scores are denoted by ' $*$ ', where $\mathrm{p}<0.05$ by $\mathrm{T}$ Test. Panels $\mathrm{b}$. and $\mathrm{c}$. show the reduction in symptom and problem scores following hyaluronic acid treatment. For each, the yellow bars represent mean improvement with the nurse-led pathway, and the purple bars represent mean improvement in the patient-led pathway.

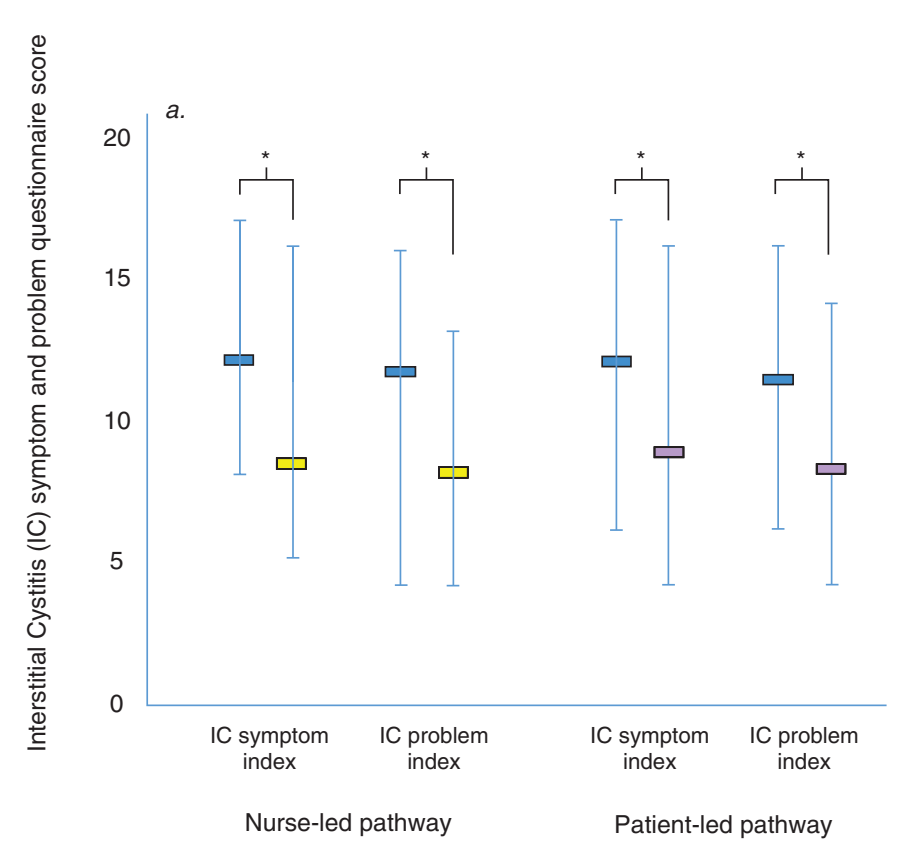

Pre-hyaluronic acid treatment
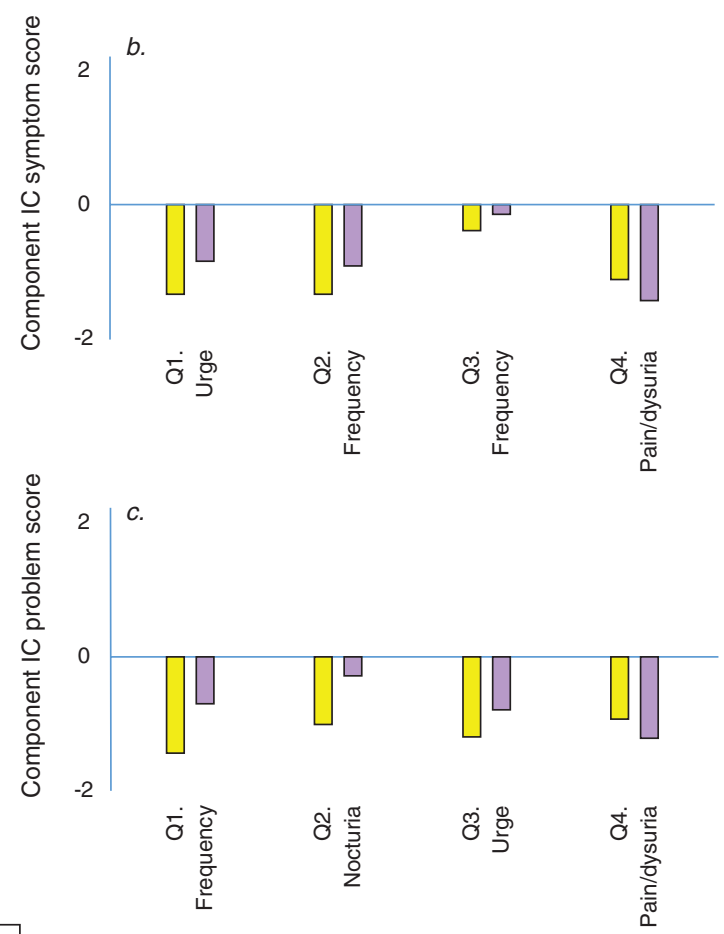

HA (Hyacyst ${ }^{\circledR} 40 \mathrm{mg}$ ) (once per week for 6 weeks then patient-led thereafter). Patients were given the option of the nurse- or patient-led pathway at the start of treatment and given the option to continue or change at each nurse clinic visit. Although, to our knowledge, there are no previous reports of similar treatment pathways for comparison, successful patientdelivered non-oral medication/treatments for chronic pain are described. ${ }^{17-19}$

Anecdotally, we observed that our patient-led pathway PBS patients were using fewer HA instillations and were requesting fewer clinic appointments, a finding substantiated by our study. Patient feedback suggested that empowerment and control over treatment timing and frequency was a driving factor in symptom control and perhaps led to the need for fewer treatments, a finding similar to that reported in other conditions causing chronic pain. ${ }^{19-22}$ Reassuringly, this empowered population also reported a significant improvement in symptoms despite fewer treatments. Indeed, the majority of our patients receiving intravesical HA had symptomatic benefit, in keeping with previous reports, ${ }^{11,12,22}$ and therefore supporting the validity of our data. No patients had problems performing intermittent self-catheterisation in the community. Interestingly, conversely to previous studies, nocturia was a difficult symptom (and problem) to improve in our case series. Although we 
FIG. 3 Comparison of treatment time and number, and number of hospital visits required, between the nurseled and patient-led intravesical hyaluronic acid pathways.

Panel a. shows the mean waiting time to start intravesical hyaluronic acid on the nurse-led (yellow bar) and patient-led (purple bar) pathways. Panel b. shows the mean number of intravesical hyaluronic acid treatments received in the first year, on the nurse-led (yellow bar) and patient-led (purple bar) pathways. Panel c. shows the mean number of nurse clinic appointments used in the first year of treatment, on the nurse-led (yellow bar) and patient-led (purple bar) pathways. Panel d. shows the mean number of clinician outpatient appointments used in the first year of treatment, on the nurse-led (yellow bar) and patient-led (purple bar) pathways. Significant differences in scores are denoted by '*', where $\mathrm{p}<0.05$ by T Test.
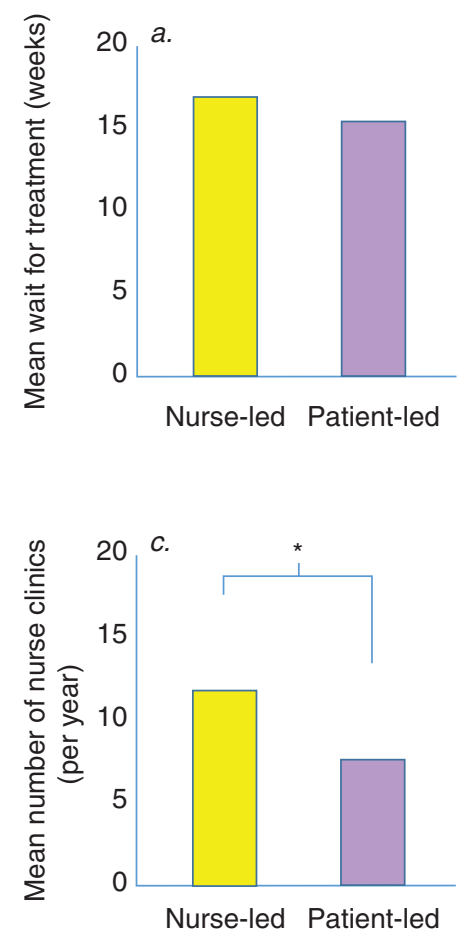
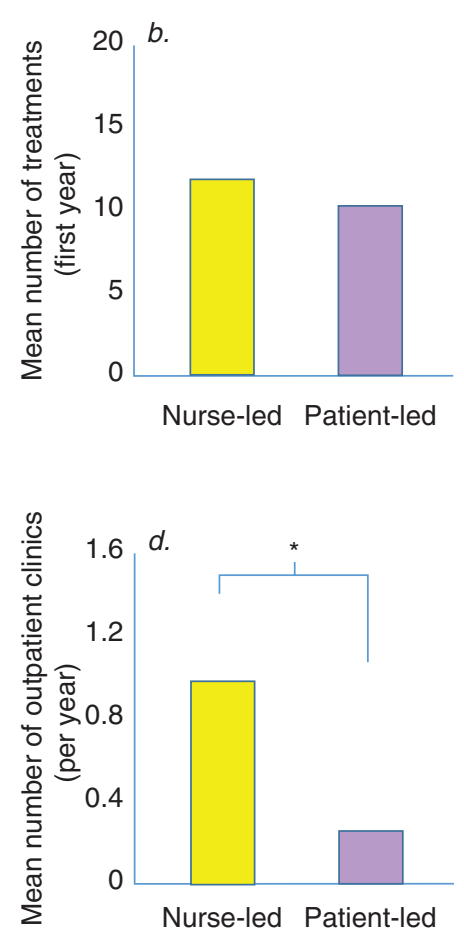

did not collect separate patient satisfaction scores, we considered improvements in ICS symptom and problem scores, and the fact that most of the patients continued HA treatment as surrogates for symptom improvement and satisfaction.

Although patient symptom benefit, empowerment and convenience are the primary reasons for this pathway, an important additional consideration is the potential benefit to service provision. In this regard, we observed that fewer nurse clinic appointments were required, fewer outpatient appointments were required, and fewer treatments were needed. Assuming, on average, 17 new patients commence (and continue) self-administered HA per year, we estimate that 76 nurse clinic appointments and 11 clinician outpatient appointments can be 'saved' per year over the nurse-led pathway. These appointments could be used for new urgent and cancer referrals and for patients requiring intra-vesical therapies for bladder cancer. Furthermore, based upon NHS National Tariff 2018/2019 figures, ${ }^{23}$ and assuming treatment continues with a constant number of hospital visits per year, we estimate that the patient-led pathway could save approximately $£ 1,500$ per patient in the first year, and approximately $£ 3,200$ for subsequent years. 
Whilst these initial analyses are promising, we do advocate caution in interpretation of our results, as we present a retrospective analysis of our (singlecentre) case series. Our patients were not randomised, the nurse-led and patient-led pathway patients were not matched nor controlled, and we recognise the possibility of inherent responder and recall bias in symptom score questionnaires. Nevertheless, given that our patient outcomes are broadly in keeping with previous reports, and the O'Leary-Sant questionnaire used is widely accepted for BPS patients, ${ }^{15,24}$ we believe our methods to be robust, and our preliminary data to be reliable and reflective of a typical UK BPS patient population.

We will continue to provide this novel service to support our local PBS patient community, and furthermore, it is our intention to develop our nursing service to offer (patient-initiated) telephone consultations, nurse-led 'at-home' community review. We hope this will be more convenient for our patients, contribute to empowerment, patient-decision making and symptom control, and relieve pressures on hospital outpatient services.

\section{CONCLUSIONS}

To our knowledge, this is the first reported case series in the UK to evaluate the use of a novel patientled at-home self-administer pathway for intravesical hyaluronic acid (HA) treatment. We have shown, in this potentially debilitating and difficult to manage spectrum of disease, that self-catheterisation and self-administration of HA is well tolerated, improves symptoms for most patients, reduces the number of patient hospital visits (and therefore the burden on nurse and outpatient clinics), and is cost-effective. Despite the limitations of our study, and although we recognise that further evaluation in greater patient numbers is required, the use of such a patient-led at-home treatment pathway should be advocated and encouraged.

No grant or funding contribution has been sought or awarded for this study

\section{ACKNOWLEDGEMENTS}

The authors would like to thank Urology Ambulatory Clinic Nurses Tracy Harrison and Michelle Bould who managed the majority of patients in the study.

\section{REFERENCES}

1. Hanno PM, Erickson D, Moldwin R, Faraday MM; American Urological Association. Diagnosis and treatment of interstitial cystitis/bladder pain syndrome: AUA guideline amendment. J Urol 2015 May;193(5):1545-53.

2. Berry SH, Elliott MN, Suttorp M, et al. Prevalence of symptoms of bladder pain syndrome/interstitial cystitis among adult females in the United States. J Urol 2011 August;186(2):540-44.

3. Hanno P, Lin A, Nordling J, Nyberg L, van Ophoven A, Ueda T, Wein A. Bladder Pain Syndrome Committee of the International Consultation on Incontinence. Neurourol Urodyn 2010;29(1):191-8.

4. Cervigni $\mathrm{M}$ and Natale F. Gynecological disorders in bladder pain syndrome/interstitial cystitis patients. Int J Urol 2014 Apr; 21 Suppl 1:85-8.

5. Nickel JC and Tripp DA; International Interstitial Cystitis Study Group. Clinical and psychological parameters associated with pain pattern phenotypes in women with interstitial cystitis/bladder pain syndrome. J Urol 2015 Jan; 193(1):138-44.

6. McKernan LC, Walsh CG, Reynolds WS, Crofford LJ, Dmochowski RR, Williams DA. Psychosocial co-morbidities in Interstitial Cystitis/Bladder Pain syndrome (IC/BPS): A systematic review. Neurourol Urodyn 2018 Mar; 37(3):926-41.

7. Grundy L, Caldwell A, Brierley SM. Mechanisms underlying overactive bladder and interstitial cystitis/ painful bladder syndrome. Front Neurosci 2018 Dec 12(12):931.

8. Hurst R, Greenwood-Van Meerveld B, Wisniewski AB, Van-Gordon S, Lin HK, Kropp BP, Towner RA. Increased bladder permeability in interstitial cystitis/ painful bladder syndrome. Transl Androl Urol 2015 Oct; 4(5):563-71. 
9. Offiah I, Didangelos A, Dawes J, et al. The expression of inflammatory mediators in bladder pain syndrome. Eur Urol 2016 Aug; 70(2): 283-90.

10. Iavazzoa C, Athanasiouab S, Pitsounia E, Falagas ME. Hyaluronic acid: an effective alternative treatment of interstitial cystitis, recurrent urinary tract infections, and hemorrhagic cystitis? Eur Urol 2007 June; 51(6): $1534-41$.

11. Lazzeri M, Hurle R, Casale P, et al. Managing chronic bladder diseases with the administration of exogenous glycosaminoglycans: an update on the evidence. Ther Adv Urol 2016 Apr;8(2):91-9.

12. Wyndaele JJJ, Riedl C, Taneja R, Lovász S, Ueda T, Cervigni M. GAG replenishment therapy for bladder pain syndrome/interstitial cystitis. Neurourol Urodyn 2019 Feb;38(2):535-44.

13. Engeler D, Baranowski AP, Berghmans B, et al. EAU Guidelines. Edn. presented at the EAU Annual Congress Barcelona 2019.

14. Han E, Nguyen L, Sirls L, Peters K. Current best practice management of interstitial cystitis/ bladder pain syndrome. Ther Adv Urol 2018 Jul; 10(7): 197-11.

15. O’Leary MP, Sant GR, Fowler FJ Jr, Whitmore KE, Spolarich-Kroll J. The interstitial cystitis symptom index and problem index. Urology 1997 May; 49(5A Suppl):58-63.

16. Jeremy Stangroom. T Test Calculator. Social Science Statistics. Available at: https://www.socscistatistics .com/tests/studentttest/default.aspx

17. Pumps. Ilias W, Le Polain B, Buchser E, Demartini L. Patient-controlled analgesia in chronic pain patients: experience with a new device designed to be used with implanted programable The oPTiMa study group. Pain Practice 2008 May/June (8);3:164-170.

18. Chakravarthy K, Chaudhry H, Williams K, Christo PJ. Review of the uses of vagal nerve stimulation in chronic pain management. Curr Pain Headache Rep Dec 2015;19:54.

19. McCorkle R, Ercolano E, Lazenby M, et al. Selfmanagement: Enabling and empowering patients living with cancer as a chronic illness. CA Cancer J Clin 2011 January/February (61);1:50-62.

20. Hoffman AJ. Enhancing self-efficacy for optimized patient outcomes through the theory of symptom selfmanagement. Cancer Nurs 2013 Jan;36(1):E16-E26.

21. Roditi D and Robinson ME. The role of psychological interventions in the management of patients with chronic pain. Psychol Res Behav Manag 2011;4:41-9.

22. Pyo JS, Cho WJ. Systematic review and meta-analysis of intravesical hyaluronic acid and hyaluronic acid/ chondroitin sulfate instillation for interstitial cystitis/ painful bladder syndrome. Cell Physiol Biochem 2016;39(4):1618-25

23. National Health System. 2017/18 and 2018/19 National Tariff Payment System. Available at: https://improvement. nhs.uk/resources/national-tariff-1719/\#h2-tariff-documents.

24. Tu FF, Kane JN, Hellman KM. Noninvasive experimental bladder pain assessment in painful bladder syndrome. BJOG 2017 Jan;124(2):283-91. 


\section{Interstitial Cystitis Symptom and Problem Questionnaire}

\section{Identifying IC}

To help your clinician determine if you have IC, please put a check mark next to the most appropriate response to each of the questions shown below. Then add up the numbers to the left of the check marks and write the total below.

IC Symptom Index

During the past month:

Q1. How often have you felt the strong need to urinate with little or no warning?

0. _ Not at all

1. - Less than 1 time in 5

2. - Less than half the time

3. - About half the time

4. - More than half the time

5. - Almost always

Q2. Have you had to urinate less than $\mathbf{2}$ hours after you finished urinating?

0 . Not at all

1. - Less than 1 time in 5

2. - Less than half the time

3. - About half the time

4. - More than half the time

5._Almost always

03. How often did you most typically get up at night to urinate?

0._None

1. Once

2. - 2 times

3. 3 times

4. 4 times

5. 5 or more times

Q4. Have you experienced pain or burning in your bladder?

0 .__ Not at all

2. - A few times

3._-Almost always

4._ Fairly often

5._Usually

Add the numerical values of the checked entries; total score:
IC Problem Index

During the past month, how much has each of the following been a problem for you?

Q1. Frequent urination during the day?
0 ._ No problem
1. - Very small problem
2. Small problem
3._- Medium problem
4._Big problem

Q2. Getting up at night to urinate?
0 ._ No problem
1._ Very small problem
2. Small problem
3._- Medium problem
4._Big problem

Q3. Need to urinate with little warning?
0 ._No problem
1._ Very small problem
2._Small problem
3._- Medium problem
4._Big problem

Q4. Burning, pain, discomfort, or pressure in your bladder?

0 ._ No problem

1.__ Very small problem

2. _ Small problem

3._- Medium problem

4._Big problem
Add the numerical values of the checked entries; total score: 